\title{
Os Sistemas BIM e a Redução de Inexatidões e Tempo nas Obras Públicas Brasileiras
}

\author{
BIM Systems and Reducing Inaccuracies and Time in the Brazilian Public Infrastructure
}

\author{
Ludmila Santos de Andrade \\ Universidade Brasília, Brasil \\ lud.de.andrade.08@gmail.com \\ Neander Furtado Silva \\ Universidade Brasília, Brasil \\ neander.furtado@gmail.com
}

\author{
Ecilamar Maciel de Lima \\ Universidade Brasília, Brasil \\ ecilamarmlima@gmail.com \\ Igor Monteiro \\ Universidade Brasília, Brasil \\ igormonteiroarqui@hotmail.com@hotmail.com
}

\begin{abstract}
The creation of an algorithm that will allow the quantitative accuracy of inputs, related to the Brazilian reality, inside the BIM platform will cause a decrease of the public costs of work. However, in this article only focus on how you can modify internally the BIM system platform for achieving the quantitative goals in planning materials. The new tool of construction planning and scheduling when using BIM systems's contribution, through the external database, will provide the construction control based on composition of inputs along the time that will allow a more economical execution to ensure the efficiency of public buildings.
\end{abstract}

Keywords: Algorithm; Accuracy of inputs; BIM; Database; Planning; Scheduling; Public buildings.

\section{Introdução}

Considerando que a construção civil é o segmento que mais consome matérias-primas e recursos naturais no planeta sendo o setor que utiliza mais de $40 \%$ da energia consumida mundialmente, contribuindo para aumentar a emissão de gases de efeito estufa, além dos custos financeiros envolvidos devido a falta de exatidão na extração de quantitativos de insumos. Por tanto, há motivos suficientes para justificar a criação de uma nova ferramenta de planejamento e programação da construção que possa minimizar tais gastos (COMPERJ, 2010).

A justificativa da existência desse trabalho é uma resposta a atual demanda do mercado e dos agentes ( profissionais,cliente) da indústria da Construção -AEC. Para que a otimização de tempo, custo, qualidade e integração das informações de projeto no campo da orçamentação possa ser simultânea no atual panorama do processo de projetação arquitetônica, a partir da utilização de plataformas de modelagem tridimensionais específicas do sistema BIM - Building information Modeling, relacionado com a realidade brasileira.

Os sistemas BIM ainda enfrentam um grande desafio na estimativa de insumos. Pois precisam ainda se interoperacionalizar com outros programas BIM de custos, como o Dprofiler, Innovaya Visual Estimating para finalizar a tarefa.

De fato a troca de informação dos sistemas BIM entre as outras plataformas ainda é problemática. A saída do modelo BIM baseado no IFC (Industry Foundation Classes) para reinterpretação em outra plataforma ainda é incompleta, por isso propomos uma nova possibilidade ao implementar o algoritmo dentro do banco de dados do BIM.

O objetivo central de grande parte dos projetos da construção civil, principalmente na esfera pública, é a execução de obras com custos mínimos orçamentários de forma a permitir a conclusão das mesmas. É necessário então a criação de uma nova ferramenta que permitira a troca de rotina dentro do banco de dados do próprio modelador. $O$ incremento de novas informações de insumos das atividades é o que buscaremos como premissa.

Para as etapas das atividades técnicas do projeto de edificação a tomada de decisão com informação mais adequada de todas as fases da obra desde sua concepção, desenvolvimento, planejamento da construção pode reduzir fortemente seus custos. A proposta então busca novas abordagens de administração de projetos para a esfera pública com a troca de rotina para a substituição do uso da porcentagem dos serviços para os quantitativos dos insumos.

A construção civil na administração pública representa investimentos de elevado valor. Por este motivo, em 1995, o Senado Federal inventariou as obras públicas federais inacabadas no Brasil. O resultado foi a identificação de 2.214 obras inacabadas que somaram o valor de $\mathrm{R} \$ 15$ bilhões de Reais desperdiçados. (BRASIL, 1995).

Todavia, este artigo mostrará como é possível modificar internamente a plataforma do sistema BIM para lograr os objetivos de troca de abordagem entre o planejamento de 
execução de porcentagens de serviços para a nova possibilidade de planejar em quantitativos de materiais. Como pode ser entendido a partir da tabela abaixo:

Tabela 1: Modelos de planejamento e programação.

\begin{tabular}{|l|c|r|rrr|}
\hline Modelo Atual: \\
\hline Quantidade de Servicos \\
\hline \multicolumn{5}{|c|}{ Pilares } \\
\cline { 1 - 3 } Formas & $\mathrm{m}^{2}$ & 66,80 & $42 \%$ & $58 \%$ & \\
\cline { 1 - 3 } Armadura & $\mathrm{kg}$ & 267,00 & $33 \%$ & $47 \%$ & $20 \%$ \\
\cline { 1 - 3 } Concreto fck 25 MPa & $\mathrm{m}^{3}$ & 4.62 & & $16.8 \%$ & $12.8 \%$ \\
\hline
\end{tabular}

\begin{tabular}{|c|c|c|c|c|}
\hline \multicolumn{5}{|c|}{ Modelo Proposto : } \\
\hline \multicolumn{5}{|c|}{ Quantidade de Insumos } \\
\hline \multicolumn{2}{|c|}{ Pilares } & & & \\
\hline Cimento & $\mathrm{m}^{3}$ & & $2,94 \mathrm{m3}$ & $1.68 \mathrm{~m} 3$ \\
\hline Areia & $\mathrm{m}^{3}$ & & $0,38 \mathrm{~m} 3$ & $0.22 \mathrm{m3}$ \\
\hline Pedra & $\mathrm{m}^{3}$ & & $0,38 \mathrm{~m} 3$ & $0,22 \mathrm{~m} 3$ \\
\hline Ferro & Barrra & $5 \mathrm{Br}$ & $4 \mathrm{Br}$ & $2 \mathrm{Br}$ \\
\hline Estribo & Barra & $7 \mathrm{Br}$ & $5 \mathrm{Br}$ & $2 \mathrm{Br}$ \\
\hline Madeira $20 \mathrm{~cm}$ & Tábuas & $\begin{array}{l}7 \\
\text { tábuas }\end{array}$ & $\begin{array}{l}4 \\
\text { tábuas }\end{array}$ & \\
\hline
\end{tabular}

\section{Problemática}

Os principais problemas na construção de obras públicas são as imprecisões de planejamento de planilha-orçamentária e de programação da execução.

O entendimento de que há etapas na construção civil que não devem ser atrasadas, sob pena de criar dificuldades ou mesmo impossibilidade no prosseguimento de outras etapas a elas ligadas. Isto quer dizer que o simples esquecimento de uma etapa pode acarretar na quebra da continuidade de toda programação de execução da obra.

O método de planejamento da obra pública se faz por porcentagem dos serviços a serem executados baseados na administração de projetos PERT/CPM (Critical Path Method). O objetivo deste método de administração de projetos é fornecer ao administrador um procedimento de racionalização da sequencia das etapas em porcentagem referentes à execução de uma obra. No entanto, este método de percentuais representa, em nosso entendimento, um equívoco, pois ao planejar a obra, se contrata por quantitativos de insumos. (BOITEUX, 1979)

Há, portanto a necessidade de criar um novo sistema que não apenas facilite o planejamento e controle de programação para a execução de obra mas principalmente permita aferir seus custos com exatidão, uma vez que todos os sistemas atuais se mostram ineficientes.

A análise da obra por meio de percentual a ser executado, ao invés de ser feita por meio de quantitativos de insumos permite que erros de cronograma ocorram. Porque ao construir se utiliza de insumos para confeccionar os serviços, e se não há um efetivo controle destes insumos ou planejamento haverá momentos em que no depósito terá excedentes ou falta de materiais.

O planejamento orçamentário da composição dos insumos (materiais, mão-de-obra e equipamentos) é um serviço de construção, que relaciona componentes adequados e indispensáveis para a sua correta execução. O uso desta abordagem permite estabelecer relações precisas entre as várias fases de uma obra.

\section{Hipótese}

Acreditamos que a criação de um algoritmo1 em uma estrutura de gestão orçamentária de quantitativos relacionados com a realidade brasileira em todo ciclo de execução da obra dentro do sistema BIM (FISCHER, 1998) oferecerá maior controle da quantidade de material usado e aquela que ainda será necessária, dentro do cronograma de tempo planejado. Possibilitando a redução de custos, precisão de insumos, e redução do tempo de construção.

$\mathrm{Na}$ indústria da construção civil a tecnologia da informação se baseia na troca de informações e contribuição no processo de projeto, com a finalidade de aumentar a o fluxo de informações a serem processadas e estas por conseguintes serem mais precisas e rápidas.

Além disso, cabe ressaltar, também, que o planejamento ineficiente da obra ocasiona atrasos de entrega de materiais resultando em custos maiores para a conclusão da obra.

Vale lembrar também que a contribuição dos sistemas BIM por meio da possibilidade de customização do banco de dados para o planejamento dos insumos para o controle das obras públicas nacionais será de extrema importância para o controle das atividades de construção pública.

Para a customização do banco de dados do sistema BIM que iniciaremos os nossos estudos por meio da formulação e resolução de problemas, bem como o uso da lógica na verificação das soluções que é o produto resultante da atividade intelectual na construção do algoritmo. Em primeiro lugar, identificaremos o problema a ser resolvido pelo algoritmo - o objetivo. Em seguida faremos a extração de todas as informações a respeito do problema (dados e operações), para relacioná-las com o conhecimento atual que se tem do assunto. Esta fase representa a definição do problema que é resultante do processo mental de abstração. (SOUZA, GOMES, et al., 2011)

\footnotetext{
${ }^{1}$ Algoritmo: conjunto ordenado e finito de etapas que permitem resolver a solução de um problema.
} 
Entendendo como resolver o problema, a próxima etapa consiste em descrever claramente os passos em uma sequencia lógica, dentro de uma convenção para que possibilite que as pessoas envolvidas na definição do algoritmo possam compreendê-lo, para solucioná-lo. (SOMMERVILLE, 1989, p. 60)

O sistema BIM no que se refere ao planejamento de quantitativos ainda não supre as exigências das obras públicas, principalmente as brasileiras devido às suas peculiaridades. Isto ocorre porque a extração automática dos sistemas BIM ainda é feita por serviços e não por quantitativos de materiais como propomos. Por, esta razão o tema abordado por este artigo é inovador e relevante ao tentar construir um algoritmo que poderia preencher esta lacuna, pois até o presente momento não existe ainda nenhuma ferramenta computacional para este fim. (DAMRONG e HADIKUSUMO, 2003)

\section{Objetivo}

Temos por objetivo criar um novo método para substituir o uso da extração automática de serviços dentro da plataforma BIM pela extração automática de quantitativos de insumos. O planejamento por quantitativos resulta em uma fiscalização mais eficiente para todos os atores da obra. (KAKU, 1998). A partir da extração automática dos insumos diretamente de uma plataforma BIM facilitará as tomadas de decisões de todas as etapas de planejamento e programação de obra.

Com isso. Pretende-se contribuir efetivamente para facilitar a execução dos serviços de obras públicas, isto é a fiscalização, medição e pagamento dos serviços, com isso poderá acarretar em uma diminuição dos custos das obras para a administração pública.

\section{Procedimetos Metodológicos}

Será necessário criar uma ferramenta externa, External Tools, e adicioná-la ao sistema BIM. Esta ferramenta será constituída por códigos da linguagem de programação orientada a objetos, para inserir esta nova tecnologia de orçamento de quantitativos internamente no sistema BIM.

O conceito da linguagem orientada a objeto começa com a abstração de dados que encapsula o processamento com dados objetos e controles de acesso aos dados e adiciona uma vinculação de método dinâmico de herança.

A herança é um conceito poderoso que destaca o potencial de reutilização do algoritmo proposto, proporcionando assim a possibilidade de significativa diminuição do tempo no desenvolvimento da ferramenta e, além disso, a possibilidade de se adicionar novas composições de serviços da obra, assim que se encontrar a necessidade de acrescentar novas atividades.

O conceito de controle de unidade de programação orientada a objeto é definido a partir da ideia de que programas simulam o mundo real. Uma vez que o mundo é preenchido por objetos reais, uma simulação do mundo então deve incluir objetos simulados. Na verdade, uma linguagem baseada nos conceitos de simulação do mundo real só precisa incluir um modelo de objetos que pode enviar e receber mensagens e reagir a estas mensagens que recebe. (SEBESTA, 2005)

A essência da programação orientada a objeto é resolver problemas. Por isto identifica-se os objetos do mundo real e os problemas para o processamento necessário desses objetos com a intenção de criar simulações dos mesmos para corrigí-los. Os processos e a comunicação necessária entre os objetos são a cadeia de informação para efetivar a simulação do ambiente (SCHNEIDER, 1999). Tipos de dados abstratos, vinculação dinâmica e herança, são os conceitos que fazem não só possível a resolução de problemas orientados a objeto, mas também convenientes e efetivos para a hipótese proposta.

\section{Plataforma Teste}

A partir da plataforma do Microsoft Visual Studio .NET e o uso da linguagem visual, a plataforma Revit .NET API (Application Programming Interface), permite que outros programas baseados no desenvolvimento de sistema. NET tenha acesso a informação do modelo gráfico e dos paramentos de informação dos objetos dentro do sistema Revit. Esta ferramenta também permite que o software matriz possa se desenvolver no conceito "third party" para que desenvolvedores construam aplicações que aumentem a produtividade do Revit

Uma interface de programa de aplicação (API) é um conjunto de rotinas, protocolos e ferramentas para a construção de aplicações de soluções de problemas. API's são mecanismos de customização que podem ser usados para aumentar as potencialidades do programa nativo sem ter a necessidade do uso de outras plataformas.

Para se ter acesso a customização da plataforma API será necessária a referência ao RevitAPI. DII e o RevitAPIUI.dll localizado no diretório de programa Revit. O RevitAPI.dll contêm os métodos usados para acessar as aplicações do Revit, como documentos, elementos e parâmetros no nível do banco de dados. O RevitAPIUI.dll contem a interface relacionada a manipulação e customização da interface do usuário (AUTODESK, 2012).

A plataforma Revit API é instalada com o Revit Architecture. A linguagem para testar o algoritmo será a C\# por ser uma Linguagem de programação compatível com a Microsoft .NET framework 4.0, além de ser uma linguagem de fácil aprendizado. O código utilizado no Visual C\# Express é um conjunto de instruções legíveis (código-fonte) que precisam ser convertidas em código que possa ser compreendido e executado por computador.

Para o teste de compilação que será executado no C\# ocorrerá isso: a informação é "empacotada", por um código executável que 
resulta em uma DLL (biblioteca de vínculo dinâmico) que poderá ser carregado no Autodesk Revit, a ferramenta externa.

\section{A conectividade do banco de dados}

A customização da plataforma BIM como se propõe só é possível por razão da existência do banco de dados do Revit que é baseado em informações externas ao sistema Open Database Connectivity (ODBC). O formato ODBC é um método de acesso ao banco de dados padrão desenvolvido pela Microsoft largamente aceito. ODBC pode ser considerado um tipo de API, oferecendo um mecanismo de fornecedor neutro para intercâmbio de dados entre aplicações, constituindo a base do sistema BIM. A proposta do ODBC é permitir a acessibilidade da informação para inúmeras aplicações BIM.

Esta vantagem externa simplifica e reduz a complexidade de encapsular a informação completa e inter-relaciona-la ao objeto dentro do modelo. Além disso, o armazenamento externo das informações no Revit possibilita que os modelos permaneçam leves e permite o acesso de informações mais rápido para aplicações externas.

\section{Conclusão}

A partir dos algoritmos obtidos verificaremos se a hipótese levantada é promissora. O uso dos sistemas BIM e dos recursos para a orientação do planejamento dos orçamentos e programação do controle de construção baseados nos insumos de composição possibilitará uma execução mais econômica para assegurar a eficiência das construções públicas, contribuindo para o incremento do número de obras concluídas.

\section{References}

AUTODESK. Revit 2012 API Developer's Guide. [S.I.]: [s.n.], 2012.

BOITEUX, C. D. Administracao de Projetos. $5^{\circ}$. ed. Rio de Janeiro: Interciência, 1979.

BRASIL, S. F. Relatório Final: o retrato do desperdício no Brasil. Comissao Temporária das Obras Inacabadas. Brasília, p. 435. 1995.

COMPERJ. Manual de Obras Públicas Sustentáveis. Agenda 21, 2010. Disponivel em: <www.agenda21comperj.com.br>. Acesso em: 10 Setembro 2013.

DAMRONG, C.; HADIKUSUMO, B. INTEGRATED 4D CAD AND CONSTRUCTION SAFETY PLANNING INFORMATION FOR A BETTER SAFETY MANAGEMENT. CAADRIA, 2003.

FISCHER, K. M. A. M. Visualization of Construction Planning Information. Automation in Construction, v. 07, n. 06, 1998.

KAKU, M. How Science Will Revolutionize the 21 Century. Anchor Book. New York. 1998.

SEBESTA, R. W. The Concepts of Programming Languages. $7^{\circ}$. ed. Colorado: Pearson Education, 2005.

SOMMERVILLE, I. Software engineering. 3. ed. Avon: Addison-Wesley Publishing Company, 1989.

SOUZA, M. F. et al. Algoritmos e Lógica de Programacao. $2^{\circ}$. ed. Sao Paulo: CENGAGE Learning, v. $1^{\circ}, 2011$. 